\title{
Generación de mapa de número de curva con sistema de información geográfica
}

\section{Generation of curve number map with geographical information system}

\author{
Marcelo Portuguez Maurtua ${ }^{1^{*}}$, Carlos Verano Zelada ${ }^{2}$ \\ ${ }^{1}$ Universidad Nacional Agraria La Molina, Perú ${ }^{2}$ Autoridad Nacional del Agua, Perú
}

\section{RESUMEN}

Recientemente, la aplicación de modelos hidrológicos ha ido en aumento por ser una herramienta efectiva que nos permite representar los diferentes procesos involucrados en la distribución de la lluvia y la generación de caudales en una determinada cuenca. Para la estimación del proceso de infiltración se han utilizado diferentes métodos, siendo uno de los modelos empíricos más extendidos y experimentados para la cuantificación de la infiltración, el de Numero de Curva del Servicio de Conservación de Suelo (SCS) de los EEUU, que es un parámetro hidrológico utilizado en la estimación de escorrentía. El objetivo de este estudio fue la generación de mapas temáticos de Número de Curva para el ámbito del territorio peruano, Esta metodología se basó en la utilización de herramientas de los Sistemas de Información Geográfica (SIG), los cuales permiten trabajar con datos diversos como mapas de cobertura y uso, textura de suelos y modelos digitales de elevaciones (MDE). Los mapas temáticos se obtuvieron mediante la aplicación de tablas de reclasificación y operaciones de superposición de las distintas capas de información de las que depende, obteniendo como resultado mapas raster de "Número de Curva", para condiciones normales, condiciones húmedas y condiciones secas, parámetros muy importantes en estudios hidrológicos.

Palabras clave: Numero de curva, curva numero, raster $\mathrm{CN}, \mathrm{CN}$ del SCS.

\begin{abstract}
Recently, the application of hydrological models has been increasing because it is an effective tool that allows us to represent the different processes involved in the distribution of rain and the generation of flows in a basin. For the estimation of the infiltration process, different methods have been used, being one of the most extended and experienced empirical models for the quantification of the infiltration, the Curve Number of the Soil Conservation Service (SCS) of the USA, which is a hydrological parameter used in the runoff estimation. The objective of this study was the generation of thematic maps of Curve Number for the area of the Peruvian territory, This methodology was based on the use of Geographic Information Systems (GIS) tools, which allow to work with diverse data such as maps of coverage and use, texture of floors and digital models of elevations (MDE). The thematic maps were obtained through the application of reclassification tables and overlapping operations of the different layers of information on which it depends, obtaining as a result raster maps of "Curve number", for normal conditions, wet conditions and dry conditions, parameters very important in hydrological studies.
\end{abstract}

Keywords: Curve number, CN raster, SCS CN.

Historial del artículo:

Recibido: 6 de junio de 2016; aprobado: 19 de junio de 2016; disponible en línea: 30 de junio de 2016

* M. Sc. en Recursos Hídricos, Ing. Agrícola UNALM, Especialista en Sistema de Información Geográfica - Universidad Distrital Francisco José de Caldas - Bogotá, Colombia- Docente de de la Facultad de Ingeniería Agrícola, Universidad Nacional Agraria La Molina.

Correo:mportuguez@lamolina.edu.pe 


\section{INTRODUCCIÓN}

Los Sistemas de Información Geográfica (SIG) nos permiten operar gran cantidad de información distribuida en el espacio con facilidad y rapidez, siendo de suma utilidad a la hora de su implementación en tareas como el proyecto y/o la investigación de temas relacionados con los recursos hídricos (Scuderi, 2006).

Un modelo hidrológico, busca representar los diferentes procesos involucrados en la distribución de la lluvia y la generación de caudales en una determinada cuenca. Una parte importante del movimiento del agua viene determinada por la infiltración, que es el proceso por el que la precipitación se mueve hacia abajo a través de la superficie del suelo, aumentando la humedad del suelo, uno de los métodos más extendidos y experimentados de los modelos empíricos de infiltración es el del SCS.

Los métodos para estimar la escorrentía a partir de la precipitación tratan de descontar de la lluvia caída sobre una cuenca todas aquellas pérdidas que se deben a factores tales como la infiltración, la evapotranspiración, la intercepción y el almacenamiento superficial. El procedimiento más generalizado y fácil de adaptar a cualquier región es el método del Número de Curva (NC), desarrollado por el Servicio de Conservación de Recursos Naturales de EE.UU. (Natural Resources Conservation Service - NRCS), originalmente llamado Servicio de Conservación de Suelos (Soil Conservation Service - SCS) en 1950, para calcular la precipitación efectiva como una función de la lluvia acumulada, la cobertura del suelo, textura del suelo y las condiciones de humedad (NRCS, 2008). Es un método empírico para el cálculo de la transformación de lluviaescorrentía, que surgió de la observación del fenómeno hidrológico en distintos tipos de suelo en varios estados y para distintas condiciones de humedad. Se observaron curvas al representarse en gráficos la profundidad de precipitación $(\mathrm{P})$ y la profundidad de exceso de precipitación o escorrentía directa $(\mathrm{Pe})$. Para estandarizar estas curvas, se definió un número adimensional de curva $\mathrm{CN}$, tal que $0<=\mathrm{CN}<=100$. Para superficies impermeables y de agua $\mathrm{CN}=100$; para superficies naturales $\mathrm{CN}<100$ y para superficie sin escurrimiento $\mathrm{CN}=0$. Los números de curva se aplican para condiciones antecedentes de humedad normales (Condición II); para condiciones secas (Condición I) o condiciones húmedas (Condición III), se calculan los números de curva equivalentes (Chow Ven, 1994).

Riccardi (2004) refiere que el método utiliza como parámetro básico el número de curva $(\mathrm{CN})$ que representa las características medias del complejo hidrológico suelo-vegetación, estando relacionado con el estado de humedad del suelo, el uso del suelo y las prácticas de manejo. La metodología del número de la curva (NC) es la más empleada para transformar la precipitación total en precipitación efectiva. De esta manera se constituye en una herramienta de gran valor para realizar estudios hidrológicos en cuencas hidrográficas, fundamentalmente cuando hay una deficiencia de registros extensos y confiables. Esta metodología requiere del conocimiento del tipo y uso de suelo de la cuenca en estudio y registros pluviográficos (Chow Ven, 1994).

El objetivo es la generación del mapa temático de Número de Curva para el ámbito del territorio peruano en condiciones normales, condiciones húmedas y condiciones secas, siguiendo la metodología del Soil Conservation Service de los Estados Unidos.

\section{Área de estudio}

El Perú se localiza en el hemisferio Occidental con relación al Meridiano de Greenwich y en el hemisferio Sur en relación a la Línea Ecuatorial. Está ubicado en la parte central y occidental de América del Sur. Tiene una extensión de 1285215,60 km² que corresponde a $0,87 \%$ de la superficie continental del planeta, el Perú se constituye en el decimonoveno país más grande del mundo, el sexto del continente americano y el tercer país más grande de América del Sur. Su localización en relación a las coordenadas geográficas es: entre los 0 응 $01^{\prime} 48^{\prime \prime}$ y los 18ㅇ $21^{\prime} 03^{\prime \prime}$ de latitud sur y los 68우 39' $27^{\prime \prime}$ y los $81^{\circ} 19^{\prime} 3405^{\prime \prime}$ de longitud oeste. El Perú es un país mega diverso, cuenta con 11 ecorregiones $y$ 84 zonas de vida de las 117 que existen en el mundo. Posee una enorme multiplicidad de paisajes debido a sus condiciones geográficas, lo que a su vez le otorga una gran diversidad de recursos naturales. En su territorio se pueden identificar tres grandes regiones, que ha sido la forma tradicional de dividirlo según sus altitudes: Costa, Sierra y Selva (MINAM, 2012).

\section{MATERIAL Y MÉTODOS}

\section{Información cartográfica}

\section{Mapa cobertura y uso}

El MINAM (2012), como ente encargado de conducir el proceso de inventario y evaluación nacional integrada de los recursos naturales y de los servicios ambientales, elaboró el Mapa de Cobertura Vegetal del Perú, el cual constituye una herramienta de gestión del patrimonio natural y a la vez de soporte en la implementación de la Política Nacional del Ambiente. Este mapa fue elaborado mediante el análisis visual de imágenes satelitales del año 2009, luego verificado en el terreno a nivel aéreo, terrestre y fluvial. La escala de interpretación o mapeo fue de $1 / 100000$, con un área mínima de mapeo de 25 ha y, excepcionalmente, de 5 ha en casos especiales. 


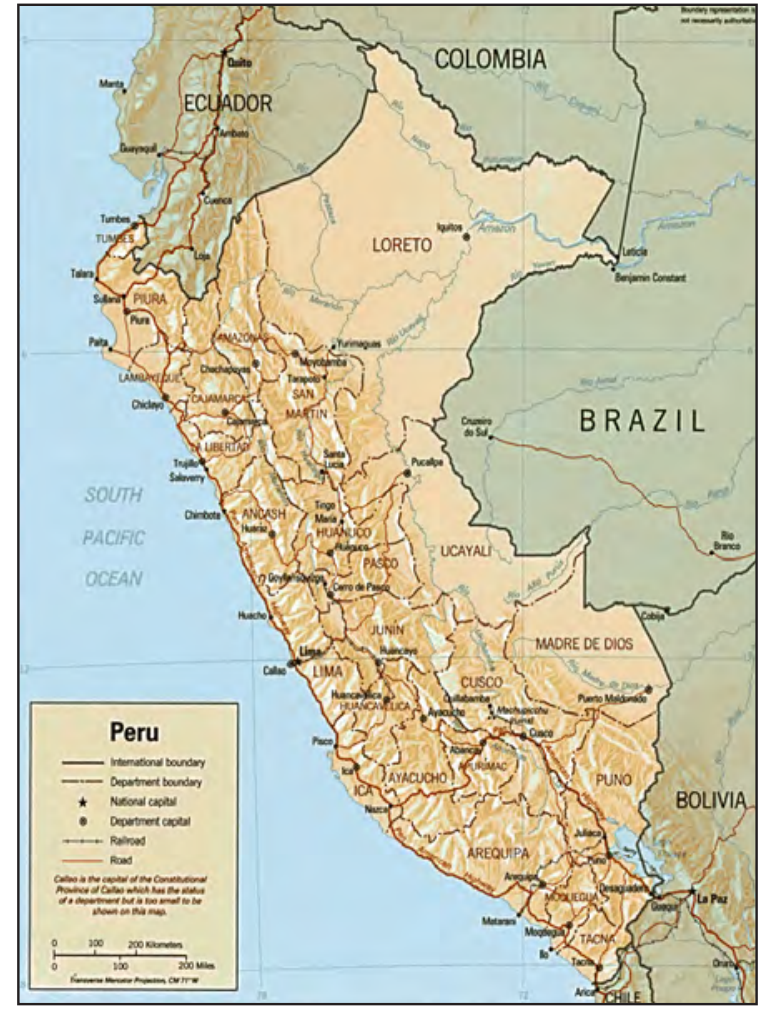

Figura 1. Imagen satelital 1,985.

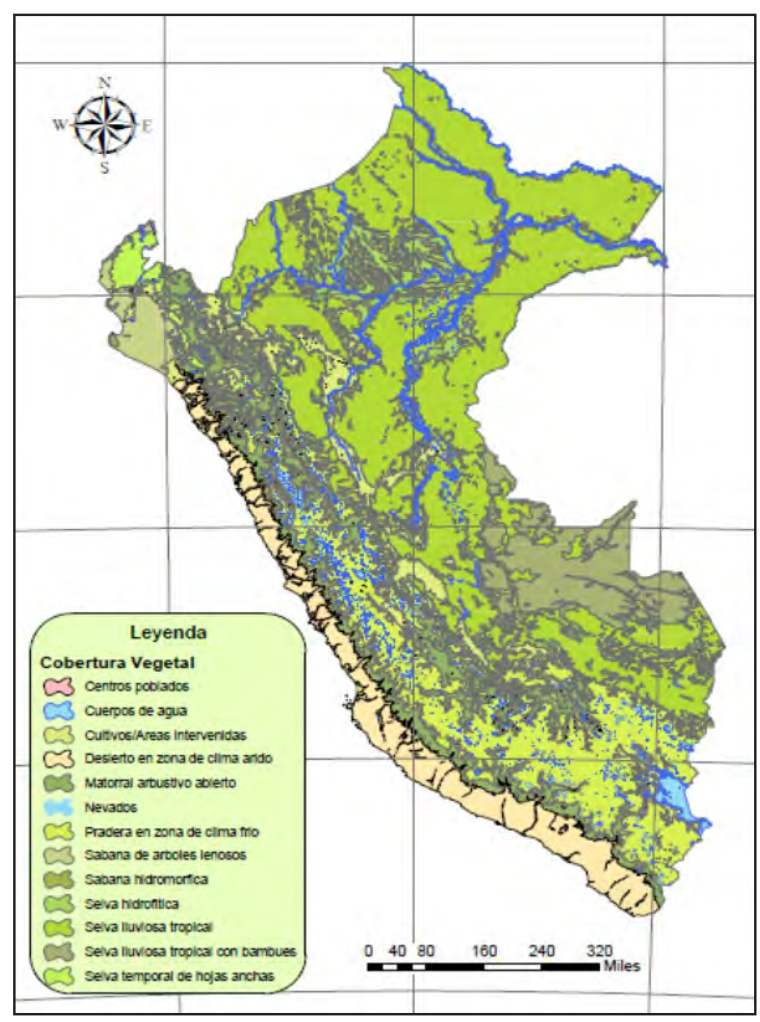

Figura 2: Mapa de cobertura vegetal.
Tabla 1. Información cartográfica.

\begin{tabular}{|c|c|c|c|}
\hline Información & Fuente & Escala & Formato \\
\hline $\begin{array}{l}\text { Cobertura } \\
\text { y uso }\end{array}$ & $\begin{array}{l}\text { Ministerio del } \\
\text { Ambiente - Perú }\end{array}$ & $1 / 100000$ & Vector \\
\hline Suelo & FAO UNESCO & 1/ 5000000 & Vector \\
\hline Topografía & $\begin{array}{l}\text { Shuttle Radar } \\
\text { Topography } \\
\text { Mission - SRTM }\end{array}$ & $\begin{array}{l}\text { Resolución } 90 \text { m } \\
\text { x } 90 \text { m }\end{array}$ & Raster \\
\hline
\end{tabular}

El mapa de cobertura (figura 2) muestra la distribución y características generales de la florística y del terreno, de los diversos tipos de cobertura vegetal que cubren el país, como, por ejemplo, los bosques lluviosos de tierra firme y pantanosos (aguajales) de la Selva Baja, los bosques lluviosos de la Selva Alta, los bosques secos del noroeste, los bosques relictos andinos, los herbazales altoandinos (pajonales, bofedales), los matorrales andinos, entre otros.

\section{Mapa de suelo}

Se trabajó con información de la FAO y UNESCO a una escala de 1:5000 000. Esta información es resultado de la colaboración de innumerables científicos de suelos en el mundo, tras un esfuerzo de 20 años. Actualmente, el Mapa Mundial de Suelos ha permanecido como la única visión global de los recursos del suelo. La figura 3 presenta el mapa de tipos de suelo para Perú.

\section{Modelo Digital de Elevaciones - MDE}

La información de elevación a escala mundial se obtuvo desde la dirección electrónica http://srtm.csi. cgiar.org/SELECTION/inputCoord.asp del SRTM, es un proyecto internacional entre la Agencia Nacional de Inteligencia-Geoespacial, NGA, y la Administración Nacional de la Aeronáutica y del Espacio, NASA ${ }^{7}$. Su fin es obtener un modelo digital de elevación de la zona del globo terráqueo entre $56 \mathrm{~S}$ a $60 \mathrm{~N}$, de modo que genere una completa base de mapas topográficos digitales de alta resolución de la Tierra. Esta base cartográfica ha sido ampliamente utilizada en diferentes campos del conocimiento relacionados con la geomática al poderse descargar gratuitamente a través de Internet. Ver figura 4 Modelo Digital de Elevación.

\section{Reclasificación de los mapas temáticos}

\section{Cobertura y uso}

A cada descripción de las coberturas se le debe asignar un código de clasificación, como se muestra en la tabla 2. 


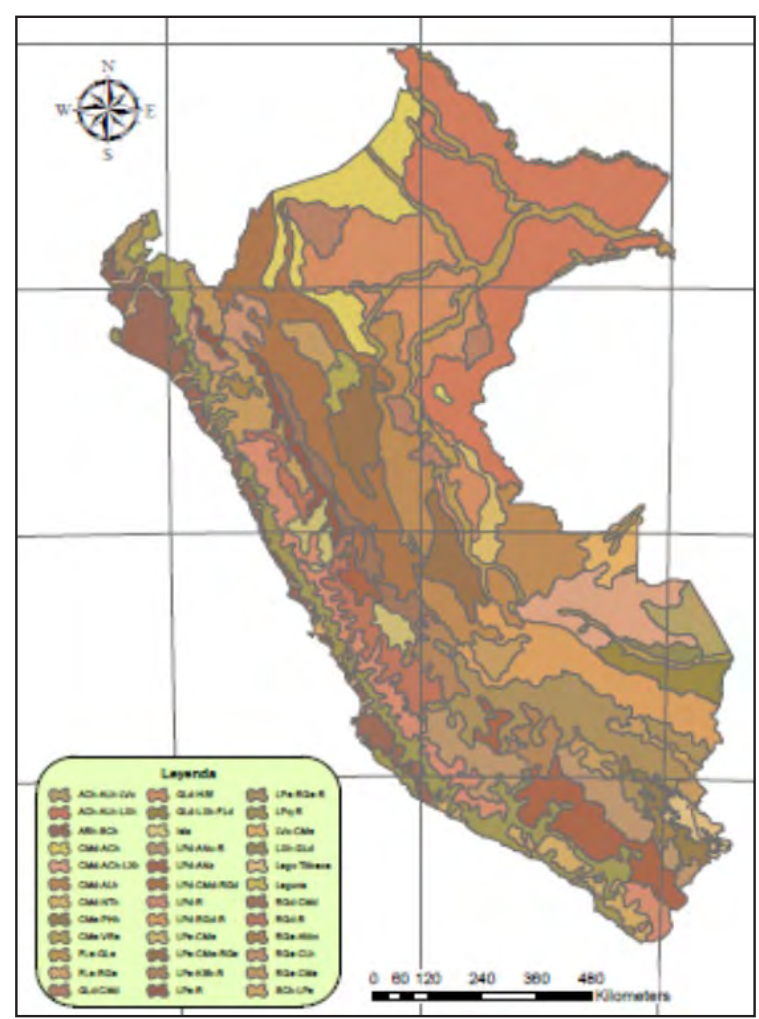

Figura 3. Mapa Temático de Suelo.

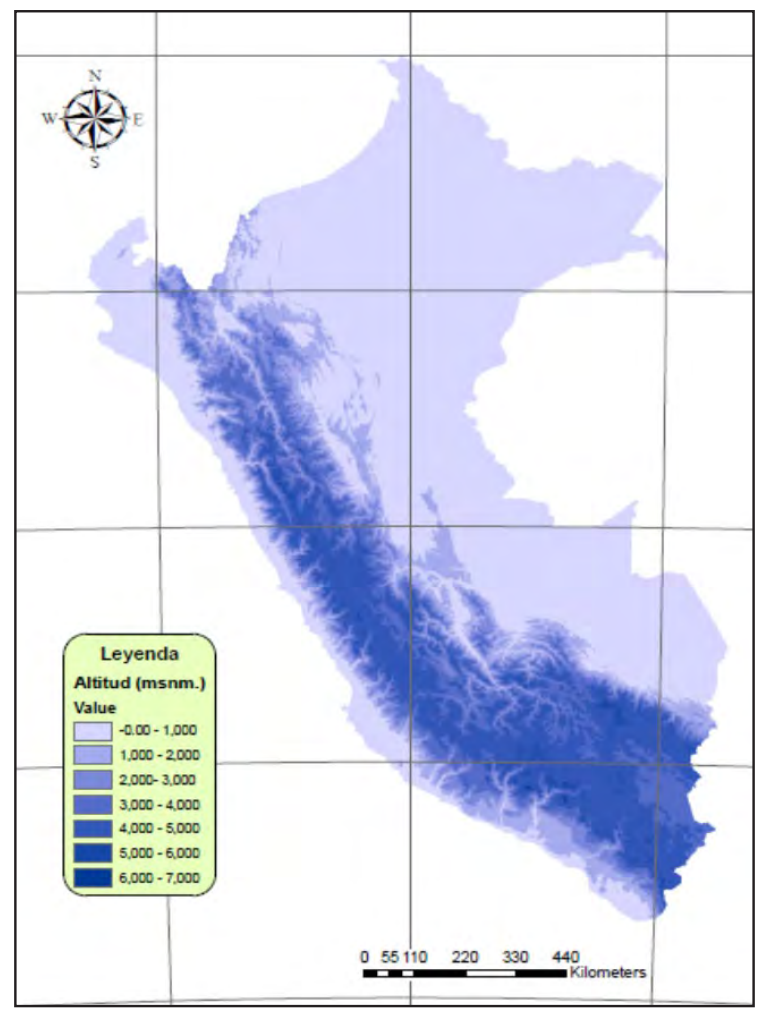

Figura 4. Modelo Digital de Elevación.
Tabla 2. Reclasificación de la cobertura y uso.

\begin{tabular}{lc}
\hline \multicolumn{1}{c}{ Descripción de coberturas } & Código \\
\hline Cuerpos de agua & 1 \\
Nevados & 2 \\
Centros poblados & 3 \\
Cultivos/Áreas intervenidas & 4 \\
Matorral arbustivo abierto & 5 \\
Pradera en zona de clima frio & 6 \\
Sabana de árboles leñosos & 7 \\
Sabana hidromorfica & 8 \\
Selva lluviosa tropical & 9 \\
Selva lluviosa tropical con bambúes & 10 \\
Selva temporal de hojas anchas & 11 \\
Selva hidrolítica & 12 \\
Desierto en zona de clima árido & 13 \\
& \\
\hline
\end{tabular}

\section{Suelo}

De acuerdo a la descripción de suelo dado por la $F A O$, se identifica a que grupo hidrológico de suelo pertenece, se muestra en la tabla 3.

\section{Características de los grupos hidrológicos de suelo}

Las características de los suelos se describen a continuación (Chow Ven, 1994):

a. En ellos el agua se infiltra rápidamente, aun cuando estén muy húmedos. Profundos y de texturas gruesas (arenosas o areno-limosas), están excesivamente drenados.

b. Cuando están muy húmedos tienen una capacidad de infiltración moderada. La profundidad de suelo es de media a profunda y su textura es francoarenosa, franca, francoarcillosa o franco-limosa. Están bien o moderadamente drenados.

c. Cuando están muy húmedos la infiltración es lenta. La profundidad de suelo es inferior a la media y su textura es franco-arcillosa, francoarcillo- limosa o arcillo-arenosa. Son suelos imperfectamente drenados.

d. Cuando están muy húmedos la infiltración es muy lenta. Tienen horizontes de arcilla en la superficie o próximos a ella y están pobremente o muy pobremente drenados. También se incluyen aquí los terrenos con nivel freático permanentemente alto y suelos de poco espesor (litosuelos).

En la tabla 4, se muestra el valor de Numero de Curva según grupo hidrológico de suelo a la que pertenecen. 
Tabla 3. Grupo hidrológico según descripción del suelo.

\begin{tabular}{|c|c|c|}
\hline Descripción de suelos & Simbología & $\begin{array}{c}\text { Grupo } \\
\text { hidrológico }\end{array}$ \\
\hline Acrisol háplico - Alisol háplico - Lixisol háplico & ACh-ALh-LXh & C \\
\hline Acrisol háplico - Alisol háplico - Luvisol crómico & ACh-ALh-LVx & C \\
\hline Arenosol háplico - Solonchak háplico & ARh-SCh & $A$ \\
\hline Cambisol dístrico - Acrisol háplico & CMd-ACh & C \\
\hline Cambisol dístrico - Acrisol háplico - Lixisol háplico & CMd-ACh-LXh & C \\
\hline Cambisol dístrico - Alisol háplico & CMd-ALh & C \\
\hline Cambisol dístrico - Nitisol háplico & CMd-NTh & C \\
\hline Cambisol éutrico - Phaeozem háplico & $\mathrm{CMe}-\mathrm{PHh}$ & B \\
\hline Cambisol éutrico - Vertisol éutrico & CMe-VRe & C \\
\hline Fluvisol éutrico - Gleysol éutrico & FLe-GLe & B \\
\hline Fluvisol éutrico - Regosol éutrico & FLe-RGe & B \\
\hline Gleysol dístrico - Cambisol dístrico & GLd-CMd & B \\
\hline Gleysol dístrico - Histosol fíbrico & GLd-HSf & $\mathrm{D}$ \\
\hline Gleysol dístrico - Lixisol háplico - Fluvisol dístrico & GLd-LXh-FLd & A \\
\hline Leptosol dístrico - Afloramiento lítico & LPd-R & B \\
\hline Leptosol dístrico - Andosol ·mbrico - Afloramiento lítico & LPd-ANu-R & B \\
\hline Leptosol dístrico - Andosol vítrico & LPd-ANz & B \\
\hline Leptosol dístrico - Cambisol dístrico - Regosol dístrico & LPd-CMd-RGd & B \\
\hline Leptosol dístrico - Regosol dístrico - Afloramiento lítico & LPd-RGd-R & B \\
\hline Leptosol éutrico - Afloramiento lítico & LPe-R & B \\
\hline Leptosol éutrico - Cambisol éutrico & LPe-CMe & C \\
\hline Leptosol éutrico - Cambisol éutrico - Regosol éutrico & LPe-CMe-RGe & B \\
\hline Leptosol éutrico - Kastanozem háplico - Afloramiento lítico & LPe-KSh-R & $\mathrm{B}$ \\
\hline Leptosol éutrico - Regosol éutrico - Afloramiento lítico & LPe-RGe-R & B \\
\hline Leptosol lítico - Afloramiento lítico & LPq-R & B \\
\hline Lixisol háplico - Gleysol dístrico & LXh-GLd & B \\
\hline Luvisol crómico - Cambisol éutrico & $\mathrm{LVx}-\mathrm{CMe}$ & C \\
\hline Regosol dístrico - Afloramiento lítico & RGd-R & B \\
\hline Regosol dístrico - Cambisol dístrico & RGd-CMd & C \\
\hline Regosol éutrico - Andosol móllico & RGe-ANm & C \\
\hline Regosol éutrico - Calcisol háplico & RGe-CLh & C \\
\hline Regosol éutrico - Cambisol éutrico & $\mathrm{RGe}-\mathrm{CMe}$ & C \\
\hline Solonchak háplico - Leptosol éutrico & $\mathrm{SCh}-\mathrm{LPe}$ & $A$ \\
\hline
\end{tabular}

Adaptación de los valores de Numero de Curva al modelo

En base de valores de los grupos hidrológicos, se elaboró la tabla 5, información que se ingresara al modelo para la generación de los mapas de Número de Curva.

Modelo conceptual

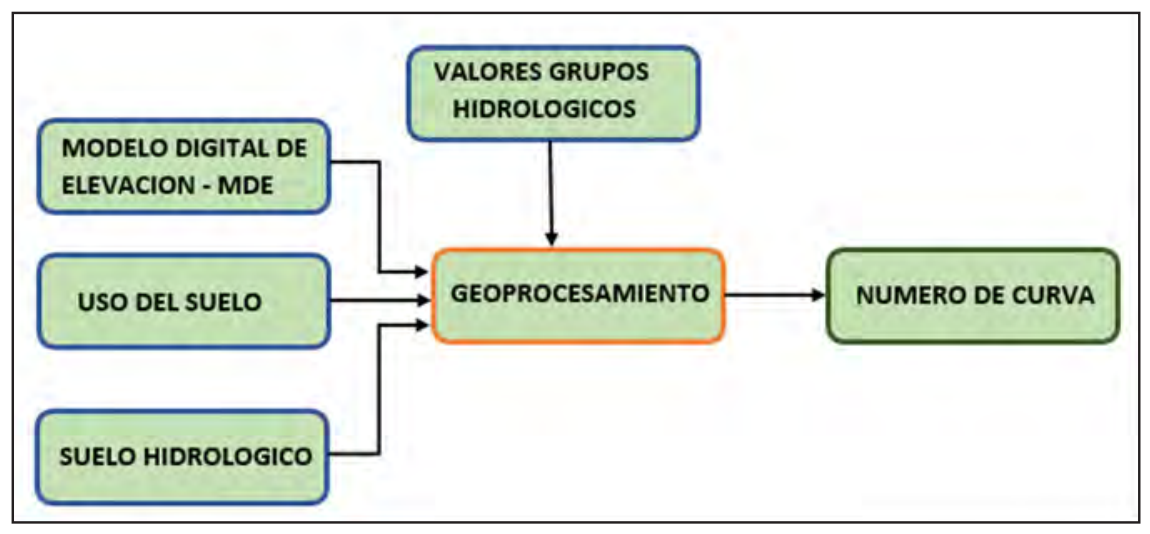

Figura 5. Modelo Conceptual. 
Tabla 4. Números de curva para distintas condiciones hidrológicas.

\begin{tabular}{|c|c|c|c|c|c|c|}
\hline \multirow{2}{*}{$\begin{array}{l}\text { Uso del suelo o } \\
\text { cobertura }\end{array}$} & \multirow{2}{*}{$\begin{array}{l}\text { Sistema de } \\
\text { laboreo }\end{array}$} & \multirow{2}{*}{$\begin{array}{l}\text { Condición } \\
\text { hidrológica para } \\
\text { infiltración }\end{array}$} & \multicolumn{4}{|c|}{$\begin{array}{l}\text { Grupo hidrológico del } \\
\text { suelo }\end{array}$} \\
\hline & & & $A$ & B & C & $\mathrm{D}$ \\
\hline \multirow[t]{2}{*}{ Barbecho } & Líneas & Mala & 77 & 86 & 91 & 94 \\
\hline & Líneas & Mala & 72 & 81 & 88 & 91 \\
\hline \multirow{5}{*}{$\begin{array}{l}\text { Cultivos } \\
\text { en línea }\end{array}$} & Líneas & Buena & 67 & 78 & 85 & 89 \\
\hline & En contorno & Mala & 70 & 79 & 84 & 88 \\
\hline & En contorno & Buena & 66 & 75 & 82 & 86 \\
\hline & En contorno/terrazas & Mala & 66 & 74 & 80 & 82 \\
\hline & En contorno/terrazas & Buena & 62 & 71 & 78 & 81 \\
\hline \multirow{6}{*}{$\begin{array}{l}\text { Cultivos } \\
\text { densos }\end{array}$} & Líneas & Mala & 65 & 76 & 84 & 88 \\
\hline & Líneas & Buena & 63 & 75 & 83 & 87 \\
\hline & En contorno & Mala & 63 & 74 & 82 & 85 \\
\hline & En contorno & Buena & 61 & 73 & 81 & 84 \\
\hline & En contorno/terrazas & Mala & 61 & 72 & 79 & 82 \\
\hline & En contorno/terrazas & Buena & 59 & 70 & 78 & 81 \\
\hline \multirow{6}{*}{$\begin{array}{l}\text { Forrajeras y } \\
\text { leguminosas } \\
\text { pasturas en } \\
\text { rotación }\end{array}$} & Líneas & Mala & 66 & 77 & 85 & 89 \\
\hline & Líneas & Buena & 58 & 72 & 81 & 85 \\
\hline & En contorno & Mala & 64 & 75 & 83 & 85 \\
\hline & En contorno & Buena & 55 & 69 & 78 & 83 \\
\hline & En contorno/terrazas & Mala & 63 & 73 & 80 & 83 \\
\hline & En contorno/terrazas & Buena & 51 & 67 & 76 & 80 \\
\hline \multirow[t]{6}{*}{ Pastura natural } & & Mala & 68 & 79 & 86 & 89 \\
\hline & & Regular & 49 & 69 & 79 & 84 \\
\hline & & Buena & 39 & 61 & 74 & 80 \\
\hline & En contorno & Mala & 47 & 67 & 81 & 88 \\
\hline & En contorno & Regular & 25 & 59 & 75 & 83 \\
\hline & En contorno & Buena & 6 & 35 & 70 & 79 \\
\hline Praderas & & Buena & 30 & 58 & 71 & 78 \\
\hline \multirow[t]{2}{*}{ Bosques } & & Mala & 45 & 66 & 77 & 83 \\
\hline & & Regular & 36 & 60 & 73 & 79 \\
\hline
\end{tabular}

Fuente: Chow et al.

Tabla 5. Valores de los grupos hidrológicos usado en el modelo.

\begin{tabular}{lrccc}
\hline \multirow{2}{*}{ Descripción del uso del suelo } & \multicolumn{4}{c}{ Grupo hidrológico } \\
\cline { 2 - 6 } & A & B & C & D \\
\hline Cuerpos de agua & 100 & 100 & 100 & 100 \\
Nevados & 98 & 98 & 98 & 98 \\
Centros poblados & 77 & 85 & 90 & 92 \\
Cultivos/Áreas intervenidas & 62 & 71 & 78 & 81 \\
Matorral arbustivo abierto & 45 & 66 & 77 & 83 \\
Pradera en zona de clima frio & 68 & 79 & 86 & 89 \\
Sabana de árboles leñosos & 45 & 66 & 77 & 83 \\
Sabana hidromorfica & 25 & 55 & 70 & 77 \\
Selva lluviosa tropical & 25 & 55 & 70 & 77 \\
Selva lluviosa tropical con bambúes & 25 & 55 & 70 & 77 \\
Selva temporal de hojas anchas & 25 & 55 & 70 & 77 \\
Selva hidrolítica & 39 & 61 & 74 & 80 \\
Desierto en zona de clima árido & 72 & 81 & 88 & 91 \\
\hline
\end{tabular}

El modelo conceptual contempla los factores que intervienen en la generación espacial de Numero de Curva (figura 5).

\section{Procedimiento para la generación de raster $\mathrm{CN}$}

Para la generación del raster de Número de Curva (CN) (Venkatesh, 2007), se utilizó la extensión HECgeoHMS para ArcGIS, el procedimiento es el siguiente:

- Con la extensión HEC -geoHMS, se debe corregir las celdas nulas o erradas del Modelo Digital de Elevación - MDE, este procedimiento es necesario realizar y eliminar cualquier error en las celdas, se usa la opción fil.

- Desde la información temática de cobertura vegetal y de suelos, se debe de acondicionar la información temática de acuerdo a las clasificaciones indicada en las tablas 2 y 3 , en seguida se realiza la superposición grafica utilizando la herramienta 
Unión, generando un nuevo mapa en formato vectorial. En este proceso en el campo FID se crearán valores -1 , esto debe ser eliminado por ser valores no intersectado.

- Utilizando la herramienta HEC-geoHMS, desde el menú Utility cargar Generate CN GRID, aparece la ventana de trabajo, en él se debe carga el MDE corregido, la información vectorial producto de la unión (mapa de cobertura vegetal y suelos), y la Tabla 5 (valores de los grupos hidrológicos), requerido para la generación espacial Numero de Curva en condiciones normales.

- Para la generación del Numero de curva para las condiciones húmedas y secas, esto se realiza en base al de condiciones normales, utilizando la herramienta mapa de algebra e ingresando las formulas 1 y 2 .

\section{RESULTADOS}

Condición de humedad antecedente, un factor importante a tener en cuenta en estas curvas son las condiciones antecedentes de humedad (Antecedent Moisture Conditions), las cuales se agrupan en tres condiciones básicas: condiciones secas (AMC I), condiciones normales (AMC II) y condiciones húmedas (AMC III). Los números de curva se aplican para condiciones antecedentes de humedad normales, y se establecen las siguientes relaciones para las otras

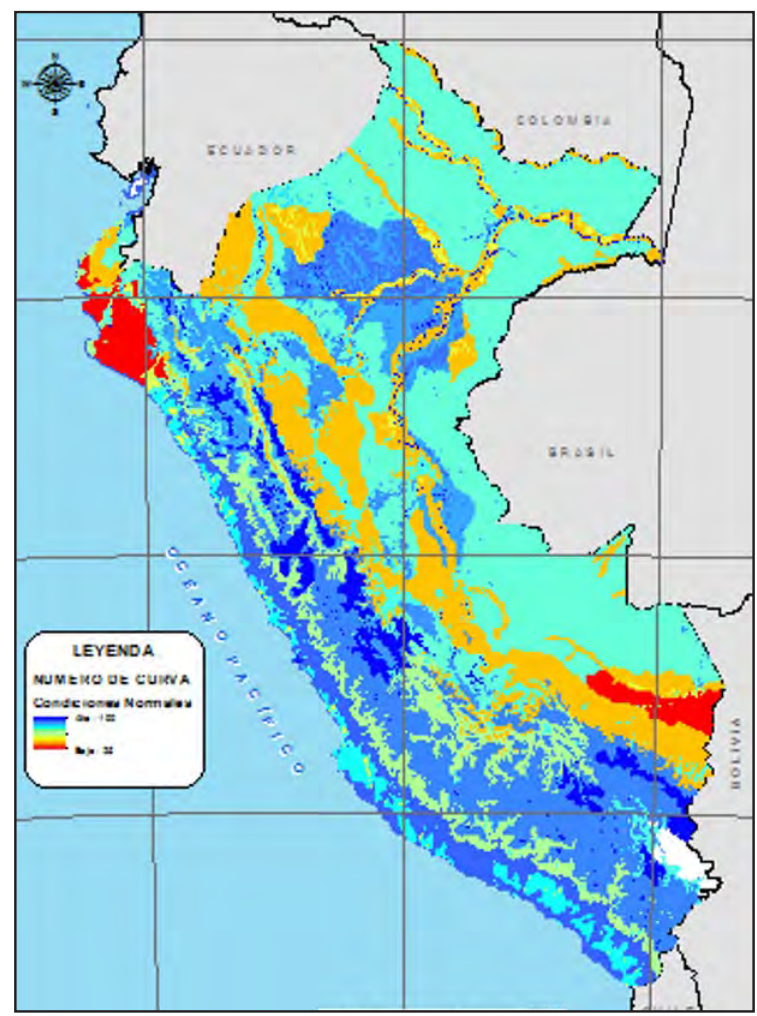

Figura 6. Mapa Numero de Curva - Condiciones normales.

$$
\begin{aligned}
& \mathrm{CN}(\mathrm{I})=\frac{4,2 \mathrm{CN}(\mathrm{II})}{10-0,058 \mathrm{CN}(\mathrm{II})} \\
& \mathrm{CN}(\mathrm{III})=\frac{23 \mathrm{CN}(\mathrm{II})}{10+0.13 \mathrm{CN}(\mathrm{II})}
\end{aligned}
$$

dos condiciones aplicando la ecuación (1) y ecuación (2) para condiciones secas y condiciones húmedas respectivamente (Chow Ven, 1994).

Para generar el mapa de Numero de Curva en condiciones normales, se realizó el análisis de geoprocesamiento, como resultado se tiene la distribución espacial de los valores de Número de Curva para condiciones Normales (figura 6).

A partir del Mapa de Numero de Curva en condiciones normales y aplicando la relación (1) y con la herramienta de Algebra de Mapas se genera Mapa de Numero de Curvas para condiciones secas (figura 7).

De modo similar que lo anterior, a partir del Mapa de Numero de Curva en condiciones normales y aplicando la relación (2) y con la herramienta de Algebra de Mapas se genera Mapa de Numero de Curvas para condiciones húmedas (figura 8).

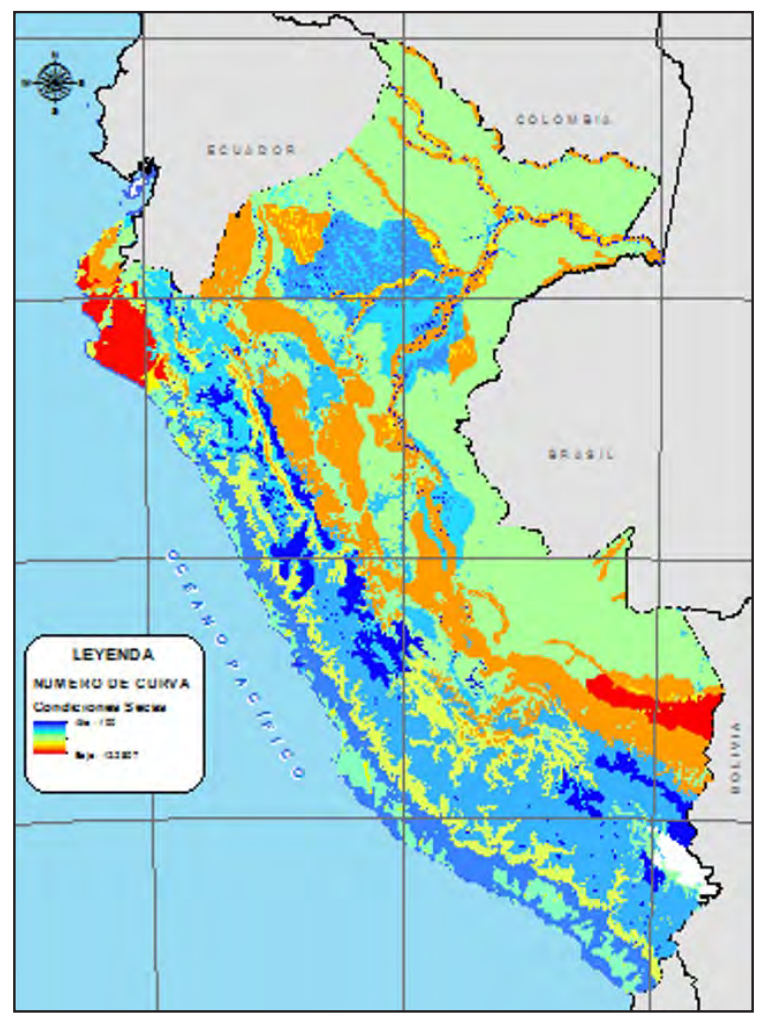

Figura 7. Mapa Numero de curva - Condiciones secas. 


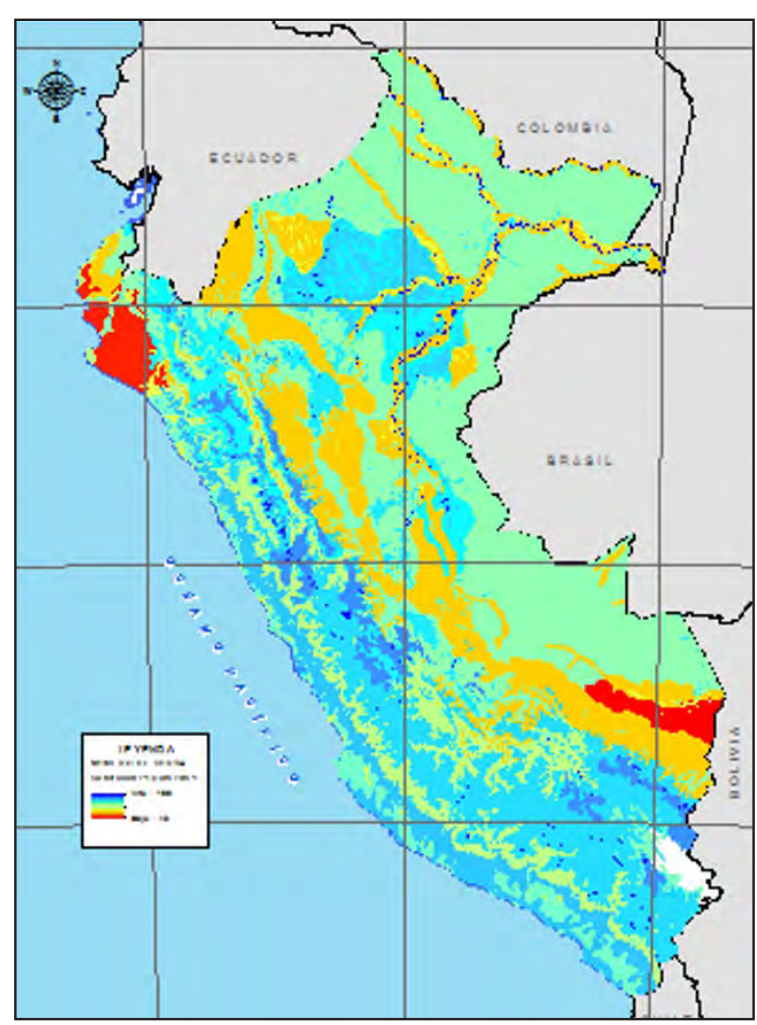

Figura 8. Mapa Numero de Curva - Condiciones Húmedas.

\section{DISCUSIÓN}

La información temática de Cobertura y uso, del total de las descripciones de cobertura en todo el ámbito del país, se agruparon en 13 clases, y a cada clase se le asignó un valor, este va desde el 1 hasta el 13 (tabla 2). De acuerdo a las características de los suelos según su descripción se le asignó al grupo hidrológico a la cual pertenece, se muestra el resultado en la tabla 3.

La tabla 4 muestra los valores de número de curva para distintas condiciones hidrológicas (fuente USBR), y en base a esta información se le asignaron valores del uso del suelo según grupo hidrológico para nuestro mapa temático, se muestra los valores en la tabla 5.

Al realizar el geoprocesamiento espacial para generar el mapa de Numero de Curva, la información final de salida es un raster, la cual en cada celda se tiene cuantificado el valor de $\mathrm{CN}$ en condiciones normales, los valores van desde 25 hasta 100 .

A partir de Mapa Numero de Curva que se generó inicialmente (Condiciones Normales), y utilizando las ecuaciones $\mathrm{CN}(\mathrm{I})$ y $\mathrm{CN}(\mathrm{III})$, y utilizando las herramientas de algebra de mapas se generaron los mapas Numero de Curva para condiciones Secas y Húmedas.

Los valores de Número de Curva para condiciones normales varían desde 25 hasta 100, para condiciones secas de 12 a 100 y para condiciones húmedas de 43 a 100 , son valores adimensionales.

Estudio realizado como Scuderi (2006), ha estimado número de curva utilizando imágenes de satélite, mediante la clasificación digital para la generación de mapa de cobertura y uso, una variable muy importante e influyente. Montserrat 10 ha utilizado tablas reclasificadas y operación de superposición de las distintas capas de información; metodología que utiliza las herramientas SIG en su proceso, una ventaja que presenta la metodología propuesta es que la actualización de los mapas del número de curva es mucho más rápida y económica puesto que supone únicamente cambiar los datos de algún mapa de variables (normalmente el de usos del suelo) y generar de nuevo el mapa de $\mathrm{CN}$, metodología similar aplicado en este estudio con la gran diferencia a una menor escala.

La generación del Mapa de Numero de Curva, está en función de los mapas temáticos de Cobertura, Suelo y Modelo Digital de Elevación, el mapa de cobertura es una de las variables más vulnerables, ya que tiene que ver con las actividades que el hombre realiza en su actividad diaria, y estos cambios de uso altera directamente a los valores adimensional de Numero de Curva que se hayan generado, motivo por lo cual se recomienda que en todo estudio hidrológico sean ajustados estos valores. En estudios hidrológicos, estos valores obtenidos (Numero de Curva) serán data de inicio, al utilizarlo en modelos hidrológicos Precipitación - escorrentía, en la fase de calibración estos valores deben ser ajustados.

Bauwe et al (2016), Petroselli et al (2013), realizaron evaluaciones hidrológicas en cuencas empleando la metodología del número de curva, juntamente a otros modelos, obteniendo buenos resultados mediante la aplicación del método de número de curva.

En conclusión, con información de mapas temáticos de cobertura y uso, mapa temático de suelo y Modelo Digital de Elevación, y en base a valores para cada grupo hidrológico de suelos teóricos, se espacializó el Numero de Curva a escala nacional, que es un insumo importante en modelos hidrológicos de Precipitación - escorrentía. La resolución espacial del Mapa de Numero de Curva es de 30 metros, a cada celda le corresponde un valor adimensional.

\section{Agradecimientos}

Los autores agradecen a la Oficina del Sistema Nacional de Información de Recursos Hídricos de la Autoridad Nacional del Agua. 


\section{REFERENCIAS BIBLIOGRÁFICAS}

Bauwe, A., Kahl, P., \& Lennartz , B. (2016). Hydrologic evaluation of the curve number and Green and Ampt infiltration methods by applying Hooghoudt and Kirkham tile drain equations using SWAT. Journal of Hydrology .

Chow , V., Maidment , D., \& Mays , L. (1994). Hidrología Aplicada. 584 . Bogotá: McGraw Hill Interamericana S.A. .

MINAM - Ministerio del Ambiente - Memoria descriptiva Mapa de Cobertura Vegetal del Perú. (Agosto de 2012).

Misión Topográfica Shuttle Radar (SRTM - Shuttle Radar Topography Mission) . (febrero de 2000).

Montserrat , F., Rodríguez , J., \& Estrela, T. (1995). Generación automática del número de curva con sistemas de información geográfica. Universidad Politécnica de Valencia. Centro de Estudios Hidrográficos, CEDEX, MOPTMA.

Natural Resources Conservation Service (NRCS). National Engineering Handbook. (2008). 630 .
Washington D.C.: Hydrology; U.S. Deparment of Agriculture.

Organización para la Alimentación y la Agricultura de Naciones Unidas (FAO). (1974). Bases de Datos Globales del Suelo .

Petroselli , A., Grimaldi , S., \& Romano , N. (2013). Curve-Number/Green-Ampt mixed procedure for net rainfall estimation: a case study of the Mignone watershed, IT. . 19, 113 - 121. Procedia Environmental Sciences .

Proyecto FAOSWALIM, Nairobi, Kenya-Universidad Mayor de San Simón. (2009). Bolivia: Guía para la descripción de Suelos - Roma.

Riccardi, G. (2004). Hidrología en Medios Antropizados. Argentina: CURIHAM, FCEIAUNR Universidad Nacional de Rosario.

Scuderi , C., \& Stenta , H. (2006). Estimación del Número de Curva (CN) A Partir de la Interpretación de Imágenes Satelitales en la Cuenca del A Ludueña, . 12, 15- 23. Santa Fe , Argentina: Cuadernos del Curiham.

Venkatesh Merwade. (2007). Creating SCS Curve Number Grid using HEC-GeoHMS . Purdue University. 